\title{
Improving Depression Screening in Primary Care: A Quality Improvement Initiative
}

\author{
Sarah R. Blackstone ${ }^{1} \cdot$ Amanda N. Sebring ${ }^{1} \cdot$ Claudia Allen $^{1} \cdot$ Joseph S. $\operatorname{Tan}^{1} \cdot$ Rebekah Compton $^{1}$
}

Accepted: 14 January 2022 / Published online: 25 January 2022

(c) The Author(s), under exclusive licence to Springer Science+Business Media, LLC, part of Springer Nature 2022

\begin{abstract}
The increase in depression during the COVID-19 pandemic underscores the importance of systematic approaches to identify individuals with mental health concerns. Primary care is often underutilized for depression screening, and it is not clear how practices can successfully increase screening rates. This study describes a quality improvement initiative to increase depression screening in five Family Medicine clinics. The initiative included four Plan-Do-Study-Act cycles that resulted in implementing a standardized workflow for depression screening, collaborative efforts with health information technology to prompt providers to perform screening via the medical record, delivering educational materials for providers and clinic staff and conducting follow-up education. Between September 2020 and April 2021 there were 23,745 clinic encounters with adult patients that were analyzed to determine whether patients were up-to-date on depression screening following their visit. A multi-level logistic regression model was constructed to determine the changes in likelihood of a patient being up-to-date on screening over the study period, while controlling for patient demographics and comorbidities. The average proportion of up-to-date patients increased from 61.03\% in September 2020 to 82.33\% in April 2021. Patients aged 65+ and patients with comorbidities were more likely to be up-to-date on screening; patients with telemedicine visits had lower odds of being up-to-date on depression screening. Overall, this paper describes a feasible, effective intervention to increase depression screening in a primary care setting. Additionally, we discuss lessons learned and recommendations to inform the design of future interventions.
\end{abstract}

Keywords Depression screening $\cdot$ Quality improvement $\cdot$ Family medicine $\cdot$ Primary care

\section{Introduction}

Major depressive disorder (MDD) affects $7-8 \%$ of adults in the US at any given time [1]. In 2020 during the COVID19 pandemic, the prevalence of adult depressive symptoms increased three-fold ( $8.5 \%$ to $27.8 \%$ ), underscoring the need for systematic approaches to identify individuals with mental health concerns [2]. In primary care, $10-14 \%$ of patients are estimated to have MDD, though as many as $50 \%$ of those go undetected in primary care [3].

Very few adults (3\%) without a diagnosis of depression are screened for depression in primary care practices [4]. To address the problem of under-recognition, the US Preventative Services Task Force and the Agency for Healthcare

Sarah R. Blackstone

bav2aw@virginia.edu

1 Department of Family Medicine, University of Virginia, PO Box 800729, Charlottesville, VA 22908-0729, USA
Research and Quality have recommended routine depression screenings for adults during primary care visits [5]. Increased secondary prevention measures for depression can improve connection to treatment and lead to better outcomes [6]. Despite these recommendations and benefits, several populations are often overlooked. Men, people over 75 years of age, minorities and uninsured patients are less likely to be screened [7]. In addition to improving screening efforts, linking patients who screen positive for depression to appropriate resources is essential. Barriers to mental health care due to provider shortages pose significant challenges [8]. Collaborative care management models involving coordination between primary care and behavioral health providers can address some of these challenges [9].

Evidence is mixed regarding the success of screening and referral programs. National record reviews have demonstrated relatively low rates of depression screening in primary care. Moreover, the usefulness of screening alone is quite limited without a treatment protocol or resources 
for patient referral [10]. Collaborative approaches such as Primary Care Mental Health Integration not only improve screening, but also increase treatment initiation and continuation $[11,12]$. Challenges to successful implementation of depression screening and follow-up include: limited visit time, lack of referral resources, results not being readily available to providers and the need to do repeat screenings $[5,13]$. Further, due to the COVD-19 pandemic, limitations on in-person office visits may hinder providers' ability to appropriately screen for depression. However, given the rise of mental health concerns associated with the pandemic, improving screening, detection and referral is of utmost importance. Barriers to addressing low depression screening rates may be mitigated by successfully incorporating EHR workflow with collaborative care models. However, it is unclear which steps can successfully promote increased depression screening among adults in primary care. To fill this gap, this study was designed to evaluate a quality improvement initiative to increase adult depression screening in five primary care clinics with Primary Care Mental Health Integration. The goal of this study was to understand how different quality improvement efforts actually impacted depression screening rates in ambulatory encounters. The specific aim of this project was to complete annual depression screenings of at least 75\% of English and Spanish-speaking patients age $\geq 18$ years. Completed annual screening was defined as completion of the Patient Health Questionnaire-2 at the office visit or within the 365 days prior to the visit. The specific questions this evaluation sought to address were:

1. Which quality improvement initiatives were associated with the greatest increase in depression screening?

2. What are the gaps in depression screening in the patient population?

\section{Methods}

\section{Study Setting}

This study took place in Charlottesville, VA in a Family Medicine Department. The Department is part of an academic medical center and has one primary clinic, with four satellite practices. Practices range in size from 2500 patients to 11,000 patients.

\section{Quality Improvement Initiative}

This project used the Plan, Do, Study, Act (PDSA) model for each quality improvement cycle. Each PDSA cycle introduced a new intervention component to support increasing depression screening.

\section{PDSA Cycle 1, September 2020}

The process began with a root cause analysis in which nursing staff were informally interviewed about perceived barriers to completing the Patient Health Questionnaire (PHQ-2), the standardized depression-screening tool. Common challenges related to a lack of standardized process for completing the PHQ-2 and reporting the results to the provider. Based on this feedback, the team developed a standardized workflow for introducing and documenting the PHQ-2, and referring patients who screen positive for depression. In collaboration with the behavioral health team, a standardized script was developed to introduce the PHQ-2 to the patient.

The depression screening process map is presented in Fig. 1. Rooming staff identified patients in need of depression screening by checking the health maintenance section in the EHR, by reviewing pre-visit documentation noted by the encounter provider, or by identifying that the patient was being seen for a Medicare annual wellness visit or hospital follow-up visit. If the patient was age $\geq 18$ years and spoke English or Spanish, the rooming staff would give the PHQ-2 and document the patient's responses in the EHR, which automatically updates the health maintenance plan. Scoring 3 or higher on the PHQ-2 indicates a need for further evaluation. Rooming staff gave these patients the PHQ-9 in their preferred language, as is standard practice in depression screening. If a patient scored 3 or greater, the patient was given the PHQ-9 and the provider was alerted. The provider assessed the patient and provided appropriate resources (see process map).

\section{PDSA Cycle 2, October 2020}

The clinic's electronic health record contains a "health maintenance" section which displays patients' status on routine screenings and provides reminders for screening to the care team. If a patient is overdue for a screening, this is flagged in the patient's chart, alerting the care team to complete the screening. As part of the initiative, the team worked with the EHR builders to including depression screening in health maintenance as a routine screening, and automatically enroll adult patients in a health maintenance reminder for annual depression screening.

\section{PDSA Cycle 3, November-December 2020}

The next PDSA cycle included an education campaign outlining the marked increase in reported depression symptoms during the COVID-19 pandemic and the importance of depression screening and early referral. These materials included the process map and goals for the quality 


\section{Depression Screening Process Map}

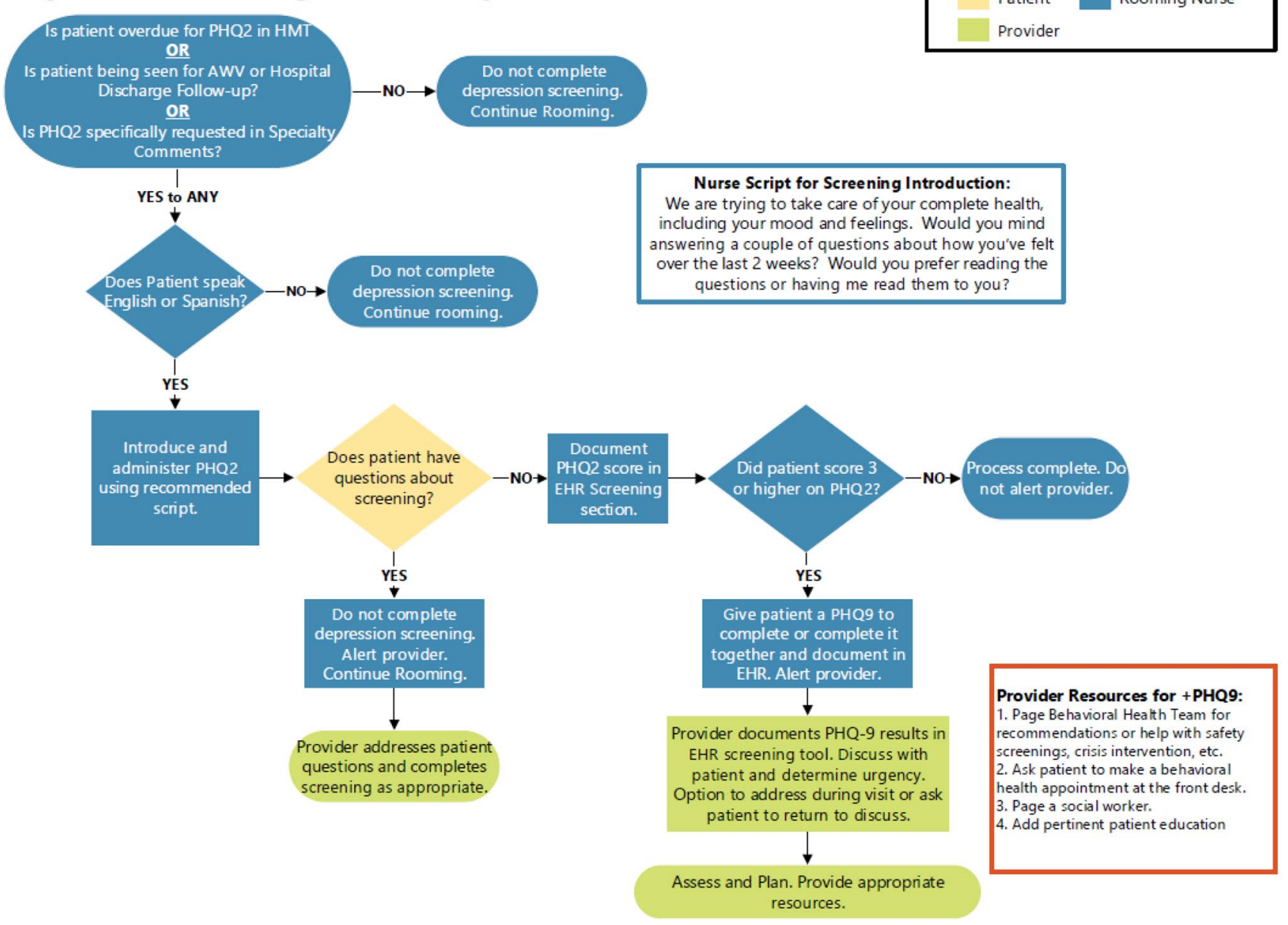

Fig. 1 Depression screening process map

improvement initiative and were shared with clinical staff and providers via email and at all-practice meetings. Providers were also asked to add a depression screening reminder to their note templates, which would prevent them from closing the encounter note until the screening was addressed. The clinic's renewed focus on improving depression screening was discussed at a patient advisory council meeting, where patient perspectives were gathered. The educational materials were presented virtually due gather restrictions resulting from the COVID-19 pandemic.

\section{PDSA Cycle 4, February 2021}

A series of email updates were sent to providers including reminders regarding the importance of depression screening, helpful tips for integrating the screening into a standard visit, and resources for screening and referring patients for depression.

\section{Data Sources}

The team used an encounter report of all clinic visits to determine the percentage of patients seen each month who were up-to-date with depression screening. These reports show a record of all clinic visits in the specified time periods. Certain types of clinic visits do not entail screening updates (e.g., nurse visits, immunization visits, medication questions, telephone questions) and were removed from the analysis. Encounter data were collected monthly between September 2020 and April 2021.

\section{Outcome Variable}

A patient's depression screening status (up-to-date versus overdue) was the study outcome variable. The following process was used to determine if patients were up-to-date on depression screening: 
1. Identify all patients enrolled in health maintenance for depression screening who are not flagged as "overdue" in the EHR (up-to-date).

2. Identify patients who are not enrolled in health maintenance for depression screening but have a PHQ-2 date documented in the last calendar year (up-to-date).

3. Identify patients who are enrolled in health maintenance for depression screening who flagged as "overdue" in the EHR (overdue).

4. Identify patients who are not enrolled in health maintenance for depression screening and do not have a PHQ-2 documented in the last year (overdue).

\section{Covariates}

Additional information was extracted from the EHR as covariates in the final analysis. These variables include age (grouped as 18-44, 45-64, 65-84, and $85+$ ) race, visit month and sex (male, female). We also explored whether have documented diagnoses of hypertension, cancer, diabetes, depression or anxiety influenced likelihood of screening. These conditions were selected based on previous literature showing increased likelihood for depression screening in patients with these diagnosis [14].

\section{Analysis}

The proportion of patients up-to-date on depression screening is presented by clinic over time. In order to simultaneously examine the associations between covariates and depression screening status, multilevel modeling (MLM) techniques were employed. The advantage of MLM is the ability to capture random variation due to multiple samples dimensions (e.g., different clinics). MLM is recommended in situations such as this as presenting aggregate data without accounting for associations at the clinic level are prone to misinterpretation. A multilevel approach takes into account the contribution of clinic-specific factors [15]. We analyzed the data with patients (level 1) nested in clinics (level 2). The high intra-class correlations (ICC $=0.45$ ) supports the use of MLM as a substantial portion of variation in outcomes is accounted for by clustering. Adjusted odds ratios are reported for the final model. Significance was determined at $\mathrm{p}<0.05$. All analyses were conducted in R.4.1.1 using the lmer package.

\section{Results}

Between September 2020 and April 2021 there were 23,745 clinic encounters examined. Majority of the patients seen were female $(64 \%)$ and white $(68 \%)$. Twenty one percent of the patients seen had a previous diagnosis of depression and $20 \%$ had a previous diagnosis of anxiety. See Table 1 for demographic information.

\section{Depression Screening Proportion}

At baseline, the proportion of adults, English or Spanish speaking patients seen each month who were up-to-date on depression screening ranged from 50 to $80 \%$ across the five clinics, with an overall department average of $61.03 \%$. In April 2021, the proportion of up-to-date patients seen ranged from 63 to $88 \%$ with a department average of $82.33 \%$. See Fig. 2.

\section{Multilevel Model}

Results of the multilevel model accounting for clinic clustering demonstrate significant increase in odds of patients being screened for depression at clinic encounters at each time point compared to baseline. Patients seen at encounters occurring in March 2021 (aOR 2.86, CI 2.54, 2.33) and April 2021 (aOR 2.79, CI 2.46, 3.17) had the greatest

Table 1 Sample demographic characteristics

\begin{tabular}{ll}
\hline Characteristic & $\mathrm{N}=23,745$ \\
\hline Sex & \\
Female & $15,265 /(64 \%)$ \\
Male & $8480 /(36 \%)$ \\
Race & \\
Non-white & $7608 /(32 \%)$ \\
White & $16,137 /(68 \%)$ \\
Age group & \\
18 to 44 & $9484 /(40 \%)$ \\
45 to 64 & $7994 /(34 \%)$ \\
65 to 84 & $5678 /(24 \%)$ \\
$85+$ & $589 /(2.5 \%)$ \\
Telemedicine visit & $2318 /(9.8 \%)$ \\
Hypertension & \\
No & $17,338 /(73 \%)$ \\
Yes & $6407 /(27 \%)$ \\
Diabetes & \\
No & $18,591 /(78 \%)$ \\
Yes & $5154 /(22 \%)$ \\
Cancer & \\
No & $23,519 /(99 \%)$ \\
Yes & $226 /(1.0 \%)$ \\
Depression & \\
No & $18,670 /(79 \%)$ \\
Yes & $5075 /(21 \%)$ \\
Noxiety & \\
Yes & $18,989 /(80 \%)$ \\
\hline & $4756 /(20 \%)$ \\
\hline
\end{tabular}


Fig. 2 Proportion of encounter patients (adult, English or Spanish speaking) up-to-date on depression screening by clinic by visit month

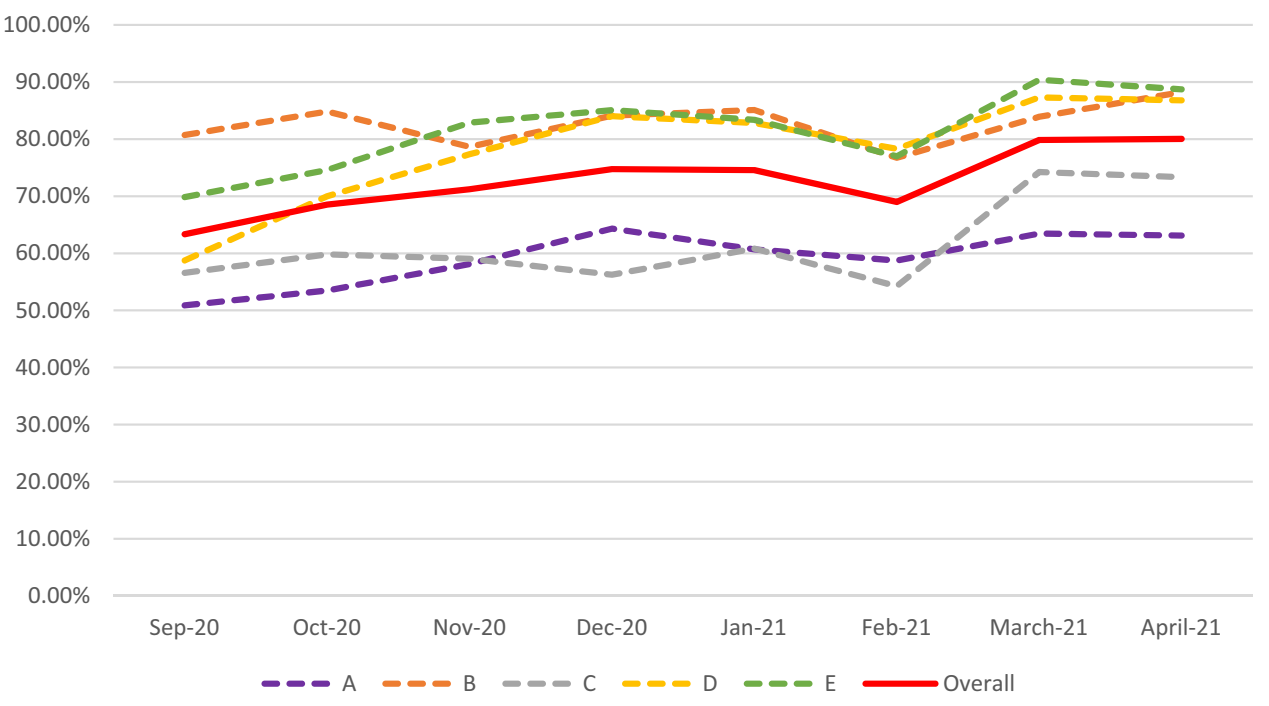

likelihood of being up-to-date on depression screening. Other factors associated with odds of being up-to-date on depression screening included age, presence of a chronic condition and type of visit (telemedicine vs. in-person).

Patients in the 65-84 age group (aOR 1.44, CI 1.32, 1.57) and in the $85+$ group (aOR 1.23, CI 1.00, 1.51) had greater odds of being up-to-date on depression screening compared to the reference group, 18-44. Individuals with hypertension (aOR 1.14, 95\% CI 1.06, 1.23), diabetes (aOR 1.21, 95\% CI 1.12, 1.32), previously diagnosed depression (aOR $1.22,95 \%$ CI 1.12, 1.32) and previously diagnosed anxiety (aOR 1.18, 95\% CI 1.09, 1.29) had greater odds of being upto-date on depression screening after the clinic encounter. Finally, individuals seen via telemedicine visits were less likely to be up-to-date on depression screening (aOR 0.51, $95 \%$ CI $0.46,0.56$ ). See Table 2 for adjusted model output.

\section{Discussion}

Systematic depression screening was successfully incorporated into a primary care setting. This QI initiative worked to improve screening rates by adopting a universal screening tool, standardizing the screening process, automating EHR workflow, educating and engaging providers and clinical staff, and developing objective criteria and processes for referral and regular data monitoring. The initiative led to an increase in depression screening at all five clinics, with a departmental improvement of $22 \%$. Between September 2020 and January 2021, overall rates of depression screening rose steadily. After the drop in screening between January 2021 and February 2021, the QI team engaged providers via regular reminders about the project importance and progress. Subsequently, we saw the greatest increase in depression screening following PDSA Cycle 4, suggesting reminders and continued team engagement are critical for sustained improvement. Prior to this, the largest increase coincided with the implementation of educational materials. Notably, these materials were presented virtually in existing practice or team meetings, which did not place additional time burdens on providers and clinic staff. Additionally, the educational materials focused on getting provider and clinic staff buy-in, to support their understanding of the importance and need for this work. The results of this study show the greatest increases in screening occurred following education and reminders, suggesting this is crucial for increasing depression screening. This study also demonstrates that feasible initiatives that can be integrated into a clinic's existing process and structure to limit the burden on staff, can lead to meaningful changes in primary care depression screening.

Despite the overall increase in depression screening, some groups of patients were less likely to be screened. Patients ages 18-44 were less likely to be up-to-date on depression screening compared to patients over 65 , despite depression being less prevalent among older adults [16]. This may be due to the requirements of Medicare Annual Wellness Visits, which include the PHQ-2. Promoting awareness about the importance of annual depression screening in all visit types may help increase screening rates in younger adults. Additionally, we found that patients with cancer, hypertension and diabetes were more likely to be up-to-date on depression screening than those without those diagnoses. Given the strong link between chronic disease and depression [17], there may be more attention to screening in this population due to increased risk. Follow-up appointments for individuals with chronic disease also present more opportunities for depression screening to occur. Similarly, patients with an anxiety or depression diagnosis were more likely to be up-to-date on screening, reflecting previous work [4]. Finally, we found that patients were less likely to be 
Table 2 Multilevel regression model results

\begin{tabular}{|c|c|c|c|}
\hline Characteristic & $\mathrm{aOR}$ & $95 \% \mathrm{CI}$ & p-value \\
\hline \multicolumn{4}{|l|}{ Visit month } \\
\hline \multicolumn{4}{|l|}{ September ${ }^{\mathrm{a}}$} \\
\hline October & 1.37 & $1.23,1.53$ & $<0.001$ \\
\hline November & 1.69 & $1.51,1.90$ & $<0.001$ \\
\hline December & 1.81 & $1.61,2.03$ & $<0.001$ \\
\hline January & 1.89 & $1.69,2.12$ & $<0.001$ \\
\hline February & 1.56 & $1.39,1.75$ & $<0.001$ \\
\hline March & 2.86 & $2.54,3.23$ & $<0.001$ \\
\hline April & 2.79 & $2.46,3.17$ & $<0.001$ \\
\hline \multicolumn{4}{|l|}{ Sex } \\
\hline \multicolumn{4}{|l|}{ Female $^{\mathrm{a}}$} \\
\hline Male & 1.00 & $0.94,1.07$ & $>0.9$ \\
\hline \multicolumn{4}{|l|}{ Age group } \\
\hline \multicolumn{4}{|l|}{18 to $44^{\mathrm{a}}$} \\
\hline 45 to 64 & 1.03 & $0.96,1.11$ & 0.4 \\
\hline 65 to 84 & 1.44 & $1.32,1.57$ & $<0.001$ \\
\hline $85+$ & 1.23 & $1.00,1.51$ & 0.048 \\
\hline \multicolumn{4}{|l|}{ Race } \\
\hline \multicolumn{4}{|l|}{ Non-White $^{\mathrm{a}}$} \\
\hline White & 0.95 & $0.89,1.02$ & 0.2 \\
\hline \multicolumn{4}{|l|}{ Telemedicine visit } \\
\hline \multicolumn{4}{|l|}{ No } \\
\hline Yes & 0.51 & $0.46,0.56$ & $<0.001$ \\
\hline \multicolumn{4}{|l|}{ Hypertension } \\
\hline \multicolumn{4}{|l|}{$\mathrm{No}^{\mathrm{a}}$} \\
\hline Yes & 1.14 & $1.06,1.23$ & $<0.001$ \\
\hline \multicolumn{4}{|l|}{ Diabetes } \\
\hline \multicolumn{4}{|l|}{$\mathrm{No}^{\mathrm{a}}$} \\
\hline Yes & 1.21 & $1.12,1.32$ & $<0.001$ \\
\hline \multicolumn{4}{|l|}{ Cancer } \\
\hline \multicolumn{4}{|l|}{$\mathrm{No}^{\mathrm{a}}$} \\
\hline Yes & 1.52 & $1.07,2.16$ & 0.019 \\
\hline \multicolumn{4}{|l|}{ Depression } \\
\hline \multicolumn{4}{|l|}{$\mathrm{No}^{\mathrm{a}}$} \\
\hline Yes & 1.22 & $1.12,1.32$ & $<0.001$ \\
\hline \multicolumn{4}{|l|}{ Anxiety } \\
\hline \multicolumn{4}{|l|}{$\mathrm{No}^{\mathrm{a}}$} \\
\hline Yes & 1.18 & $1.09,1.29$ & $<0.001$ \\
\hline Department.sd_(Intercept) & 0.51 & & \\
\hline $\mathrm{R}^{2} \mathrm{M}$ & 0.06 & & \\
\hline $\mathrm{R}^{2} \mathrm{C}$ & 0.13 & & \\
\hline
\end{tabular}

${ }^{\mathrm{a}}$ Reference category

up-to-date on depression screening at telemedicine visits. Possible reasons could include connection and set up challenges resulting in shorter visit time, lack of intake nursing staff who systematically begin the screening, or patient/provider discomfort with telemedicine platforms making them more reticent to ask/answer sensitive questions. Despite research demonstrating feasibility of telemedicine for treatment of depression [18], there is limited information about how real-time depression screening via video conferencing works. Instead, there are emerging data suggesting utility of depression screening using smartphone apps $[19,20]$.This may be an area for future consideration if gaps in telemedicine depression screening persist.

The lessons learned from this initiative reflect those of similar QI initiatives, namely the challenge in integrating depression screening into busy clinical schedules [21]). While documentation of screening in the EHR offers an advantage for data management and reporting, it can be challenging for providers to complete all required population health screenings in addition to addressing the reason for visit. The US is experiencing a primary care shortage, and by 2033 , the expected deficit is 55,000 primary care providers [22]. As this shortage continues, the challenges noted in this study may be exacerbated; however, the need for regular screening and population health management remains. Primary care practices will have to develop innovative ways to combat this challenge. One option is to leverage electronic patient portals to conduct previsit screenings, reducing the documentation burden on clinic staff during visits. Several studies have demonstrated that patient portals can be an effective platform for conducting previsit screenings or engaging patients in decisions about screenings [23, 24, 25. Prior to joining a telemedicine video call, patients could receive a link to complete the necessary screenings, allowing more time to address the reason for visit. Patient portal screenings can also benefit patients who have depression and require regular monitoring. A study using electronic patient portals for depression management demonstrated that younger patients were less likely to drop out of depression care management, a population that historically has a greater attrition rate [26]. In this study, patients under age 65 had lower rates of depression screening compared to those 65 and over; utilizing previsit screening via patient portals may help increase depression screening rates in this population [26]. While there are benefits to patient portal use, there are disparities in use and enrollment that merit consideration [27-29]. Previsit screening via patient portals may not capture all patients seen at the clinic; however, increasing previsit screening completion will decrease documentation burden on clinic staff, allowing time to complete and document screenings for patients who could not complete them prior to their visit.

\section{Limitations}

This study was limited in several important ways. The data collection for this project was solely based on EHR reports, which relies on the provider or clinical staff documenting the PHQ results into the EHR. Anecdotal evidence indicated, 
in some circumstances, that the rooming staff completed the PHQ on paper, but that the provider did not enter the results into the EHR. Thus, the data captured from the EHR may not accurately reflect depression screening at each time point. Due to the number of encounters during the study period, there were not resources to conduct chart review for each encounter in which patients were not up-to-date on their depression screening per the EHR. This challenge underscores the importance of not only having a standardized process, but developing new strategies for obtaining screening data (e.g., previsit screening via MyChart) that does not require additional provider time. Another limitation to this study is the inclusion of only English and Spanish speakers. The study team made this decision because of inconsistent evidence demonstrating the validity of the PHQ-2 in other languages commonly represented in the clinics (e.g., Dari, Swahili). Thus, the generalizability of these results to other patient populations is limited. Finally, as part of this study we did not assess whether patients who screened positive for depression had sufficient follow-up including visits with a mental health provider. The QI initiative detailed a comprehensive referral plan, including a warm hand-off to integrated, on site behavioral health. However, many patients who screen positive for depression during routine appointments are referred to mental health care providers in the community, which are not linked with the health system EHR. While we are able to determine if and when patients referred within the health system see a behavioral health provider, we are not able to assess this for the majority of patients who are referred outside the health system. Therefore, these results reflect the screening process only and not patient follow-up.

\section{Conclusions}

This study showed significant improvement in depression screening over an eight-month QI initiative; patients seen for visits in later study months had greater odds of being upto-date on annual depression screening compared to patients seen in the baseline month. Future research is needed to assess whether the referral loop is closed for patients who screen positive; this may involve collaborating with external clinics to integrate patient billing information into the referring organization's EHR. Additionally, there is a need for developing innovative ways to complete population health management screenings without increasing the documentation burden on clinic staff, such as exploring mechanisms of conducting electronic, pre-visit screenings.

Author Contributions RC, JT and CA conceptualized the study. AS developed the evaluation plan. SB performed the literature review and statistical analysis. Both AS and SB drafted the manuscript. RC, JT and CA provided feedback on the manuscript. All authors reviewed and approved the final version.

Data Availability The data that support the findings of this study are available from the corresponding author upon request.

\section{Declarations}

Conflict of interest We have no funding or competing interests to disclose.

Ethical Approval This study received exemption from the Health Sciences Research Institutional Review Board as a Quality Improvement project.

Informed Consent This study received exemption from consent as there was no patient interaction and the study was conducted as part of a Quality Improvement initiative.

\section{References}

1. Hasin, D. S., Sarvet, A. L., Meyers, J. L., Saha, T. D., Ruan, W. J., Stohl, M., \& Grant, B. F. (2018). Epidemiology of adult DSM-5 major depressive disorder and its specifiers in the United States. JAMA Psychiatry, 75(4), 336-346. https://doi.org/10.1001/jamap sychiatry.2017.4602

2. Ettman, C. K., Abdalla, S. M., Cohen, G. H., Sampson, L., Vivier, P. M., \& Galea, S. (2020). Prevalence of depression symptoms in US adults before and during the COVID-19 pandemic. JAMA Network Open, 3(9), e2019686-e2019686. https://doi.org/10.1001/ jamanetworkopen.2020.19686

3. Pignone, M., Gaynes, B., Rushton, J., Mills-Burchell, C., Orleans, T., Mulrow, C., \& Lohr, K. (2002). Screening for depression in adults: a summary of the evidence for the U.S. Preventive Services Task Force. Annals of Internal Medicine, 136(10), 765-776. https://doi.org/10.7326/0003-4819-136-10-200205210-00013\% m12020146

4. Bhattacharjee, S., Goldstone, L., Vadiei, N., Lee, J. K., \& Burke, W. J. (2018). Depression screening patterns, predictors, and trends among adults without a depression diagnosis in ambulatory settings in the United States. Psychiatric services, 69(10), 1098-1100.

5. Park, L. T., \& Zarate, C. A. (2019). Depression in the primary care setting. New England Journal of Medicine, 380(6), 559-568. https://doi.org/10.1056/NEJMcp1712493

6. Pfoh, E. R., Janmey, I., Anand, A., Martinez, K. A., Katzan, I., \& Rothberg, M. B. (2020). The impact of systematic depression screening in primary care on depression identification and treatment in a large health care system: A cohort study. Journal of General Internal Medicine, 35, 3141-3147.

7. Kato, E., Borsky, A. E., Zuvekas, S. H., Soni, A., \& Ngo-Metzger, Q. (2018). Missed opportunities for depression screening and treatment in the United States. The Journal of the American Board of Family Medicine, 31(3), 389-397.

8. Thomas, K. C., Ellis, A. R., Konrad, T. R., Holzer, C. E., \& Morrissey, J. P. (2009). County-level estimates of mental health professional shortage in the United States. Psychiatric services, 60(10), 1323-1328.

9. Unützer, J., Katon, W., Callahan, C. M., Williams, J. W., Jr., Hunkeler, E., Harpole, L., Hoffing, M., Della-Penna, R. D., Noël, P. H., Lin, E. H., Areán, P. A., Hegel, M. T., Tang, L., Belin, T. R., 
Oishi, S., \& Langston, C. (2002). Collaborative care management of late-life depression in the primary care setting: a randomized controlled trial. JAMA, 288(22), 2836-2845.

10. Bergus, G. R., Hartz, A. J., Noyes, R., Jr., Ward, M. M., James, P. A., Vaughn, T., Kelley, P. L., Sinift, S. D., Bentler, S., \& Tilman, E. (2005). the limited effect of screening for depressive symptoms with the PHQ-9 in rural family practices. The Journal of Rural Health, 21(4), 303-309.

11. Bohnert, K. M., Pfeiffer, P. N., Szymanski, B. R., \& McCarthy, J. F. (2013). Continuation of care following an initial primary care visit with a mental health diagnosis: Differences by receipt of VHA Primary Care-Mental Health Integration services. General Hospital Psychiatry, 35(1), 66-70.

12. Szymanski, B. R., Bohnert, K. M., Zivin, K., \& McCarthy, J. F. (2013). Integrated care: Treatment initiation following positive depression screens. Journal of General Internal Medicine, 28(3), 346-352.

13. Colligan, E. M., Cross-Barnet, C., Lloyd, J. T., \& McNeely, J. (2020). Barriers and facilitators to depression screening in older adults: A qualitative study. Aging \& mental health, 24(2), 341-348.

14. Akincigil, A., \& Matthews, E. B. (2017). National rates and patterns of depression screening in primary care: Results from 2012 and 2013. Psychiatric Services, 68(7), 660-666.

15. Weinmayr, G., Dreyhaupt, J., Jaensch, A., Forastiere, F., \& Strachan, D. P. (2017). Multilevel regression modelling to investigate variation in disease prevalence across locations. International Journal of Epidemiology, 46(1), 336-347.

16. Fiske, A., Wetherell, J. L., \& Gatz, M. (2009). Depression in older adults. Annual Review of Clinical Psychology, 5(1), 363-389. https://doi.org/10.1146/annurev.clinpsy.032408.153621

17. Chapman, D. P., Perry, G. S., \& Strine, T. W. (2005). The vital link between chronic disease and depressive disorders. Preventing Chronic Disease, 2(1), A14.

18. García-Lizana, F., \& Muñoz-Mayorga, I. (2010). Telemedicine for depression: A systematic review. Perspectives in Psychiatric Care, 46(2), 119-126. https://doi.org/10.1111/j.1744-6163.2010. 00247.x

19. BinDhim, N. F., Shaman, A. M., Trevena, L., Basyouni, M. H., Pont, L. G., \& Alhawassi, T. M. (2015). Depression screening via a smartphone app: Cross-country user characteristics and feasibility. Journal of the American Medical Informatics Association, 22(1), 29-34. https://doi.org/10.1136/amiajnl-2014-002840

20. Kim, J., Lim, S., Min, Y. H., Shin, Y. W., Lee, B., Sohn, G., Jung, K. H., Lee, J. H., Son, B. H., Ahn, S. H., Shin, S. Y., \& Lee, J. W. (2016). Depression screening using daily mental-health ratings from a smartphone application for breast cancer patients. Journal of Medical Internet Research, 18(8), e216. https://doi.org/10. 2196/jmir.5598
21. Corathers, S. D., Kichler, J., Jones, N.-H.Y., Houchen, A., Jolly, M., Morwessel, N., Crawford, P., Dolan, L. M., \& Hood, K. K. (2013). Improving depression screening for adolescents with type 1 diabetes. Pediatrics, 132(5), e1395-e1402. https://doi.org/10. 1542/peds.2013-0681

22. Medicine, A. C. o. S. (2020). ACSM Position Stands. Retrieved from https://www.acsm.org/acsm-positions-policy/official-posit ions/ACSM-position-stands

23. Bose-Brill, S., Feeney, M., Prater, L., Miles, L., Corbett, A., \& Koesters, S. (2018). Validation of a novel electronic health record patient portal advance care planning delivery system. Journal of Medical Internet Research. https://doi.org/10.2196/jmir.9203

24. Krist, A. H., Woolf, S. H., Hochheimer, C., Sabo, R. T., Kashiri, P., Jones, R. M., Lafata, J. E., Etz, R. S., \& Tu, S.-P. (2017). Harnessing information technology to inform patients facing routine decisions: cancer screening as a test case. The Annals of Family Medicine, 15(3), 217-224.

25. Woolf, S. H., Krist, A. H., Lafata, J. E., Jones, R. M., Lehman, R. R., Hochheimer, C. J., Sabo, R. T., Frosch, D. L., Zikmund-Fisher, B. J., \& Longo, D. R. (2018). Engaging patients in decisions about cancer screening: Exploring the decision journey through the use of a patient portal. American Journal of Preventive Medicine, 54(2), 237-247. https://doi.org/10.1016/j.amepre.2017.10.027

26. Pecina, J., North, F., Williams, M. D., \& Angstman, K. B. (2017). Use of an on-line patient portal in a depression collaborative care management program. Journal of Affective Disorders, 208, 1-5. https://doi.org/10.1016/j.jad.2016.08.034

27. Ancker, J. S., Barrón, Y., Rockoff, M. L., Hauser, D., Pichardo, M., Szerencsy, A., \& Calman, N. (2011). Use of an electronic patient portal among disadvantaged populations. Journal of General Internal Medicine, 26(10), 1117-1123. https://doi.org/10. 1007/s11606-011-1749-y

28. Goel, M. S., Brown, T. L., Williams, A., Hasnain-Wynia, R., Thompson, J. A., \& Baker, D. W. (2011). Disparities in enrollment and use of an electronic patient portal. Journal of General Internal Medicine, 26(10), 1112-1116. https://doi.org/10.1007/ s11606-011-1728-3

29. Sarkar, U., Karter, A. J., Liu, J. Y., Adler, N. E., Nguyen, R., López, A., \& Schillinger, D. (2011). Social disparities in internet patient portal use in diabetes: Evidence that the digital divide extends beyond access. Journal of the American Medical Informatics Association, 18(3), 318-321. https://doi.org/10.1136/ jamia.2010.006015

Publisher's Note Springer Nature remains neutral with regard to jurisdictional claims in published maps and institutional affiliations. 